\title{
Comercialización de la agricultura familiar para el desarrollo rural: feria de la Universidad Nacional de La Matanza
}

\author{
Marketing of family farming for rural development: fair of the National \\ University of La Matanza
}

\author{
Maria Carolina Feito ${ }^{1}$ (1) \\ ${ }^{1}$ Consejo Nacional de Investigaciones Científicas y Técnias (CONICET), Buenos Aires, Argentina. E-mail: carofeito@gmail.com
}

\begin{abstract}
Cómo citar: Feito, M. C. (2020). Comercialización de la agricultura familiar para el desarrollo rural: feria de la Universidad Nacional de La Matanza. Revista de Economia e Sociologia Rural, 58(1), e187384.

https://doi.org/10.1590/1806-9479.2020.187384
\end{abstract}

\begin{abstract}
Resumen: Mostramos la importancia de los canales alternativos de comercialización del sector de la Agricultura Familiar como herramienta para el desarrollo rural, relevando una experiencia de comercialización alternativa para productores familiares de La Matanza, provincia de Buenos Aires Argentina: la "Feria del Productor al Consumidor: la soberanía alimentaria entra a las aulas", en la Universidad Nacional de La Matanza. Aplicamos metodología cualitativa con trabajo de campo etnográfico (entrevistas a feriantes, extensionistas, visitantes y consumidores, y observaciones realizadas durante ediciones de la feria y reuniones de la asamblea de feriantes). También utilizamos datos secundarios de fuentes bibliográficas y estadísticas nacionales sobre agricultura familiar y ferias en Argentina. Debatimos sobre desarrollo rural en Argentina y las funciones estratégicas de la comercialización en la AF. Describimos la situación de las ferias de la AF en Argentina y analizamos la experiencia presentada. Concluimos que este sector productivo, a pesar de su relativa informalidad, debe ser considerado por su aporte económico al desarrollo nacional y su rol fundamental de proveedor de alimentos al mercado interno. Estos agricultores requieren políticas diferenciales que consideren sus particulares características. Por otra parte, reconocemos que estas ferias contribuyen al desarrollo rural como potente instrumento de comercialización alternativa para el sector, mejoran intercambios entre actores, reflejado en el incremento del capital social.
\end{abstract}

Palabras clave: comercialización, agricultura familiar, desarrollo rural.

Abstract: We show the importance of alternative marketing channels of the Family Farming (FF) sector as a tool for rural development, revealing an alternative marketing experience for family producers in La Matanza, province of Buenos Aires, Argentina: the "Producer Consumer Fair: food sovereignty enters the classroom", at the National University of La Matanza. We apply qualitative methodology with ethnographic field work (interviews with traders, extension agents, visitors and consumers, and observations made during editions of the fair and meetings of the assembly of traders). We also use secondary data from bibliographic sources and national statistics on FF and fairs in Argentina. We discuss rural development in Argentina and the strategic functions of commercialization in the FF. We describe the situation of the FF fairs in Argentina and analyze the experience presented. We conclude that this productive sector, despite its relative informality, must be considered for its economic contribution to national development and its fundamental role as a provider of food to the domestic market. These farmers require differential policies that consider their particular characteristics. On the other hand, we recognize that these fairs contribute to rural development as a powerful alternative marketing instrument for the sector, improve exchanges between actors, reflected in the increase in social capital.

Keywords: marketing, family farming, rural development. 


\section{Introduccción}

Analizamos una experiencia de comercialización alternativa a los mercados convencionales concentradores, para productores de la agricultura familiar (en adelante, AF) del partido de La Matanza, provincia de Buenos Aires, desarrollada mediante una estrategia de articulación entre distintos tipos de actores: una institución académica (la Universidad Nacional de La Matanza - Unlam); otra de intervención en el ámbito agropecuario (la Estación Experimental Area Metropolitana de Buenos Aires del Instituto Nacional de Tecnología Agropecuaria - EEA AMBA INTA) y varias organizaciones de productores locales. Se trata de la "Feria del Productor al Consumidor: la soberanía alimentaria entra a las aulas". Se realizaron desde fines del año 2015 hasta la fecha, ocho ediciones de dos días consecutivos entre semana, con un total de ocho horas diarias de exposición y venta de productos, en el predio de la sede San Justo de la Universidad, ubicado a unos $17 \mathrm{~km}$ de la Ciudad Autónoma de Buenos Aires. Nuestro objetivo es mostrar la importancia de los canales alternativos de comercialización de la Agricultura Familiar como herramienta para el desarrollo rural de Argentina, especialmente a nivel local, mostrando la importancia de este sector agropecuario para la economía nacional, como actor productivo y en su rol de proveedor de alimentos para el mercado interno. Utilizamos metodología cualitativa con trabajo de campo etnográfico, basado en entrevistas a los feriantes, técnicos extensionistas, visitantes y consumidores, así como observaciones, durante varias ediciones de la Feria y participando en reuniones de la asamblea de feriantes, realizadas en el mismo predio de la Universidad. Realizamos análisis de datos secundarios de fuentes bibliográficas y estadísticas nacionales sobre agricultura familiar y ferias en Argentina.

Lo rural no es únicamente lo agrario, sino "un espacio donde reside la población y se desarrollan actividades que trascienden la producción agropecuaria" (Lattuada et al., 2012, p. 173). Por eso son vitales los vínculos rural-urbanos y la promoción de actividades y servicios no agropecuarios relacionados con las particularidades y recursos de cada región o localidad. Hay que considerar esto para una política para el desarrollo con eje en lo productivo, para lograr la transformación económica, social e institucional de las áreas rurales. Esta política debe ser construida junto a los actores relevantes, en una articulación entre lo local y lo global, y vinculando cuatro dimensiones articuladas por lo productivo: humana y social; territorial; competitividad, tecnología e innovación (acceso a mercados dinámicos); reglas de juego institucionalizadas (sumar actores privados a estrategia incluyente de sectores económicos más débiles y vulnerables, promovida desde el Estado). La intervención estatal debe ser de carácter redistributivo, para el que mejore sea el sujeto de la política diferencial.

El desarrollo rural es público y también privado, ya que requiere políticas sectoriales y territoriales activas y diferenciales, articuladas con políticas macro para el bienestar del conjunto de la sociedad rural. Estas políticas no deberían ser exclusiva responsabilidad estatal, se requiere la inclusión de organizaciones de la sociedad civil. Nuevas condiciones institucionales requieren capacidad técnica y profesional instalada en territorio, grado de motivación y una importante masa de organizaciones con experiencia previa (Lattuada et al., 2012).

La AF es un sector productor de alimentos de proximidad, pero para que éstos lleguen en tiempo y forma a los consumidores, se requieren canales alternativos de comercialización, así como promover el consumo de alimentos sanos, producidos cuidando el medioambiente, y fortalecer el asociativismo de los productores. Esto amerita considerar la interconexión entre las distintas esferas del sistema agroalimentario: producción, comercialización y consumo. El periurbano es un territorio estratégico para el desarrollo de sistemas de suministro de alimentos de proximidad. Es por esto que seleccionamos para el análisis, una feria realizada en el marco de una institución universitaria, como canal alternativo para que los alimentos producidos por la AF puedan proveer seguridad alimentaria a los consumidores locales.

Trabajamos los conceptos de: i) desarrollo rural como construcción social y la generación de esta problemática en Argentina; ii) la AF como categoría en construcción, mostrando que la necesidad de conceptualizar desde el Estado este sector conllevó una cierta tipificación de la categoría, así como la manera en que se define este sujeto de políticas diferenciales; iii) la comercialización desde el enfoque de tramas de valor, mostrando la importancia de las tramas institucionales (conformadas por organismos públicos y privados dirigidas a 
organizaciones de pequeños productores para favorecer su inclusión económica y social), para implementar procesos de desarrollo rural; iv) la cuestión alimentaria como una cuestión de Estado.

Finalmente, identificamos herramientas para formulación e implementación de políticas públicas diferenciales para este sector, particularmente para promover y reforzar los espacios comerciales alternativos a los tradicionales.

\section{Metodología}

Utilizamos un abordaje cualitativo, conocido como enfoque etnográfico, centrado en el estudio de narrativas y prácticas de los sujetos y la reconstrucción de sus trayectorias de vida (Geertz 1992; Guber 1991), ya que dicho enfoque busca comprender los fenómenos sociales desde la perspectiva de sus miembros. Se realizó trabajo de campo etnográfico (Guber 1991, p. 83), aplicando la "no directividad" a través de técnicas no invasoras, en un intento de progresivamente "ampliar la mirada" y la capacidad de detectar y registrar información significativa, bajo el supuesto de que aquello de orden subjetivo es sumamente significativo del comportamiento objetivo, y aplicando la reflexividad del investigador (quien no conoce situándose externamente a su objeto de conocimiento, sino ubicándose en una relación activa con lo que se propone conocer). La Unidad de Estudio (ámbito espacial donde se realizó la investigación), es la Feria del Productor al Consumidor en el predio de la Universidad Nacional de La Matanza. La Unidad de Análisis (los sujetos de la investigación) fueron los feriantes que participaron en distintas ediciones de la Feria.

La metodología cualitativa se sustenta en: (i) información primaria: sistematización de entrevistas semi-estructuradas y de entrevistas y observaciones no estructuradas a informantes calificados; (ii) información secundaria (de instituciones locales, documentación bibliográfica y estadísticas nacionales, provinciales y municipales).

Ander Egg (1995) sostiene que

en ciencias sociales, casi siempre se requiere estudiar algún problema relacionado con un sector muy amplio de personas o cosas, que por múltiples razones no es posible llegar a todos y a cada uno de sus elementos; en tales casos se recurre a la selección de una parte, bajo ciertas condiciones, sobre la cual se trabajará en la investigación, es decir se utiliza una muestra (Ander Egg, 1995, p. 91).

Taylor \& Bodgan (1987) aluden a que la muestra de los investigadores cualitativos evoluciona conforme avanza su estudio. Por su parte Glasser \& Strauss (1967) se refieren a un muestreo teórico que se basa en la selección consciente de casos adicionales con el fin de desarrollar nuevas intelecciones o ampliar las ya existentes. Coincidiendo con Guber (2001), resulta insuficiente emplear la muestra probabilística para comprender la dinámica social, modos organizativos, sentidos por los que se orientan sus miembros, especialmente los inesperados o desconocidos por el investigador. Esto se debe, en parte, a que la muestra probabilística se suele aplicar a partir de categorías y criterios preestablecidos por el investigador. Es por esto que decidimos utilizar el otro tipo de muestra más frecuente en la investigación antropológica que es la no probabilística. En primer término, utilizaremos la "muestra de oportunidad", basada en la autoselección de los informantes y en que un individuo se ofrece a dar información e incluso llega a colaborar como recolector de información. La ocasión y la eventualidad, la oportunidad del encuentro y el "caerse bien" entre el informante y el investigador, son un requisito importante. Los marcos de la selección en este tipo de muestra están definidos por criterios sumamente flexibles y se van delineando conforme avanza la investigación, la comunicatividad con los informantes, la claridad y la amplitud de la mirada del investigador. La muestra de oportunidad demanda del investigador una mayor apertura para detectar qué atributos son social y culturalmente relevantes, y cuáles no lo son. Además, complementaremos este tipo de muestra con la muestra intencional propuesta por Martinez Carazo (2006), quien explica que se suelen eliminar los casos atípicos o muy peculiares y calibrar la influencia de todo lo que tiene carácter excepcional; sin embargo, se procura que la muestra represente lo mejor posible los subgrupos naturales y que se complementen y 
equilibren recíprocamente. Es decir, se tratará de buscar una muestra que sea comprehensiva y que tenga, a su vez, en cuenta los casos negativos, pero haciendo énfasis en los casos más representativos y paradigmáticos y explotando a los informantes clave. En conclusión, el investigador tratará de imitar al buen fotógrafo, que busca los mejores ángulos para capturar la mayor riqueza de la realidad que tiene delante. Escogeremos dos criterios para la selección de la muestra: el muestreo por cuotas que tiene como finalidad "representar al total de la población a través del establecimiento de sectores proporcionales o cuotas que reflejen las diferentes categorías que son características en el universo que se vaya a estudiar" (Ander Egg, 1995, p. 92). Es decir, el investigador de acuerdo a su experiencia y conocimiento de la población, establece cuotas o sectores con la cantidad de casos que en número sean representativos de la población a estudiar. El muestreo por cuotas se complementará con el muestreo bola de nieve que se logra a través de amigos, parientes, contactos personales y conocidos como se accede a capturar los actores objeto de la investigación. Consistirá en una serie limitada de entrevistas o grupos de hablantes extremos (sirven para contar con los rasgos o conductas límites de una clase o grupo), ejemplares (se utilizan para visualizar ciertas características ya conocidas) o típicos (permiten la descripción de los rasgos de los sujetos más repetidos de una población caracterizada por una homogeneidad interna) en relación a ciertas prácticas sociales (Serbia, 2007, citado en Martinez Carazo, 2006). Nuestra muestra consistió en una selección de feriantes que participan regularmente de la Feria analizada, determinados por los criterios precedentemente expuestos.

Respecto de las técnicas cualitativas aplicadas, la técnica de la entrevista no estructurada es la más apropiada para acceder al universo de significación de los actores, comienza en la búsqueda de preguntas y sentidos, utilizando los procedimientos: atención flotante del investigador; asociación libre del informante; categorización diferida posterior del investigador. La observación y la participación son dos vías específicas y complementarias de acceso a lo real: la observación requiere un grado mínimo de participación para obtener información significativa. Para el registro se realizó reconstrucción escrita a posteriori de cada sesión de campo, mediante anotaciones en libreta de campo, grabaciones, videos y fotografías, confección de fichas temáticas y de informantes a través de planillas de observación, lo cual permitió reconstruir con alto grado de detalle la trama social, identificando y diferenciando distintas posturas adoptadas por los sujetos sociales frente a la temática estudiada. Se realizaron entrevistas en profundidad a los feriantes, técnicos extensionistas, visitantes y consumidores durante la realización de las distintas ediciones de la Feria y observaciones participantes en las ediciones de la feria y en reuniones de feriantes e investigadores, realizadas en la Universidad. El trabajo de campo se realizó en cuatro ediciones de esta feria, entre abril de 2016 y noviembre de 2016, además de contactos fuera del espacio de la feria con productores feriantes y técnicos del Instituto Nacional de Tecnología Agropecuaria que participan de la organización de la misma.

Asimismo, utilizamos información secundaria proveniente de fuentes bibliográficas y estadísticas nacionales sobre la agricultura familiar y sobre las ferias en Argentina, relevadas por el Instituto Nacional de Estadística y Censos y por el Instituto Nacional de Tecnología Agropecuaria.

\section{Fundamentación teórica}

\subsection{El concepto de desarrollo como una construcción social}

El concepto de desarrollo fue revisado desde diversas perspectivas durante la última década del siglo XX, intentando relacionar el fracaso de políticas y proyectos de desarrollo, sus prejuicios culturales y las simplificaciones y sobreentendidos. Se entremezclaron en estas definiciones sobre desarrollo dos connotaciones diferentes: el proceso histórico de transición hacia la economía capitalista y el aumento de calidad de vida, buscando mejorar los indicadores de bienestar material (Ferguson, 1990). La evidencia histórica muestra que la relación entre ambos fenómenos es insostenible, porque el proceso de modernización desplegado en la última mitad de ese siglo extendió pobreza y marginación social en 
Latinoamérica. La fetichización del concepto de desarrollo actúa como un poderoso filtro intelectual de nuestra percepción del mundo contemporáneo (Viola Recasens, 2000). En la mayoría de documentos de instituciones de desarrollo se encuentran los prejuicios de economicismo (que identifica desarrollo y crecimiento económico por la influencia de la teoría económica neoclásica en la configuración de ideas dominantes del desarrollo) y eurocentrismo (modelo occidental como parámetro universal para medir atraso o progreso), ignorando que gran parte de la actividad económica productiva de esta región se realiza fuera del mercado (trabajo doméstico, actividades agropecuarias de subsistencia, sector informal, mediante relaciones de reciprocidad e intercambio) y desconoce todo lo que no se pueda contabilizar (desigualdad social, ecología, diversidad cultural, discriminación de género, etc).

El concepto de desarrollo presupone una determinada concepción de la historia de la humanidad, de las relaciones entre hombre y la naturaleza, asumiendo al mismo tiempo un modelo implícito de sociedad considerado como universalmente válido y deseable (Viola Recasens, 2000).

A partir de los años '70, estas expectativas de un progreso acumulativo universal se desmoronan, cuando

a cambio de imágenes culturalmente establecidas (construidas por hombres y mujeres concretos en sus espacios locales), a cambio de mitos concretos (verdaderamente reales), se ofreció al hombre moderno una expectativa ilusoria, implícita en la connotación de desarrollo y en su red semántica: crecimiento, evolución, maduración, modernización (Esteva, 2000, p. 98).

Comenzaron entonces a forjarse otras formas de pensar y representar Latinoamérica sin buscar ya un "desarrollo alternativo" sino "alternativas al desarrollo" o un "posdesarrollo", corriente que formula una sistemática deconstrucción del concepto de desarrollo (Viola Recasens, 2000). Arturo Escobar (1995) en un intento innovador, sostuvo que el discurso del desarrollo permitió la invención del Tercer Mundo en tanto categoría monolítica, ahistórica y esencialista, convirtiéndose en una nueva forma de autoridad que presentada como un conocimiento técnico, permite a las instituciones internacionales de desarrollo diagnosticar los problemas de los países más pobres, así como justificar su intervención sobre ellos.

La llegada del siglo XXI no presenta esa homogeneización a escala mundial propuesta por el concepto clásico de desarrollo. Las instituciones internacionales comienzan entonces a valorar la diversidad cultural, incluyendo la "dimensión cultural del desarrollo" como una variable esencial de cualquier proyecto.

Kottak (2000) aplica al desarrollo la regla de que la meta de la estabilidad es el principal empuje para el cambio, refiriendo el desarrollo a una evolución socioeconómica planificada, y asegurando que la gente no va a cooperar con proyectos que les exijan cambios mayores en sus vidas cotidianas, particularmente si interfieren con su forma tradicional de asegurarse la subsistencia diaria. Por eso deben tomarse en cuenta los valores culturales y conductales de la gente, que suelen diferir de aquellos de los planificadores (aprender, progresar, incrementar conocimientos técnicos, mejorar eficiencia). Propone como exitosos aquellos proyectos que tienen la meta de "cambiar para mantener", preservando los sistemas pero haciéndolos funcionar mejor, promoviendo cambios pero no sobreinnnovación. A la inversa, los proyectos que fracasan no tienen en cuenta la cultura local y suponen una sobreinnovación (proyectos de reconversión productiva que no consideran derechos tradicionales sobre tierras o la organización tradicional local). Estos proyectos suelen tener una visión de la economía como esfera separada, más que parte del sistema sociocultural local. Es decir, no justificar el cambio en función de metas abstractas, sino tomar la percepción de las necesidades locales y tratar de moldear las condiciones locales para seguir la plantilla prefijada, en lugar de intentar encajar los cambios con las necesidades locales. Por ello, la planificación del desarrollo rural requiere trabajo de campo, visitando las localidades y conversando con la gente afectada.

Dadas estas consideraciones, entendemos por desarrollo no sólo un desarrollo económico que permita aumentar ingresos y mejorar condiciones materiales de vida, sino una idea integral que considere todos los aspectos de la sociedad y la cultura, permitiendo a su vez potenciar sus capacidades existentes (Feito, 2014). 


\subsection{El desarrollo rural en Argentina}

A pesar de que Argentina es un país de base agropecuaria, la problemática del desarrollo rural no se generalizó hasta épocas recientes, tanto por preeminencia de la región pampeana sin presencia campesina relevante, como - por la ignorancia en el plano nacional de la situación de las economías regionales con realidades campesinas (especialmente en las regiones Noroeste, Noreste y Cuyo), ya sea por el escaso peso económico para el funcionamiento de la economía en su conjunto, como por el hecho de que los reclamos y necesidades de los actores de estas economías no alcanzaron un lugar en la agenda pública nacional (Lattuada et al., 2012).

El tema del desarrollo rural se instala definitivamente en los años 90, ante el diagnóstico de que la mitad de las explotaciones agropecuarias del país desaparecerían dadas las condiciones socioeconómicas impuestas por el modelo neoliberal. La concentración del poder económico producido por la apertura y desregulación del modelo, profundizó los problemas de equidad estructural. Sin embargo, coincidimos con Lattuada et al. (2012) en que no se construyó ni consolidó una institucionalidad eficiente y adecuada al nuevo escenario. Las alternativas institucionales y de gestión para el desarrollo rural no respondieron ni se adecuaron a las transformaciones socioeconómicas resultantes de los procesos de globalización y reestructuración operados en la década del 90, y cuando lo hicieron, fue en forma despareja (Manzanal, 2006). La fortaleza fue la creación de un capital social y cultural en el nivel territorial previamente inexistente, basado en la integración progresiva de una red de miles de productores nucleados en pequeños grupos y de técnicos y profesionales formados en el terreno sobre cuestiones referidas al desarrollo rural.

A partir de la crisis de los años 2001-2002, comienza en Argentina una lenta recuperación de una mayor presencia e intervención del Estado, mediante iniciativas aún poco coordinadas y sin un programa institucionalizado que organice el accionar del conjunto de agencias y funcionarios que participan en la construcción de la política agraria. Durante décadas, "desarrollo rural" fue sinónimo de "pobreza rural", tanto en el ámbito de las políticas públicas, como en los ámbitos de la academia y de la cooperación internacional. Hoy desde la academia se lo entiende desde una contribución al "empoderamiento" de pequeños productores, hasta un arma colonial para consolidar asimetrías de poder estructurales. Algunas variantes intermedias lo acotan a su carácter de instrumento para mejorar las condiciones de vida de la población rural en el marco de estados democráticos que regulan economías capitalistas (Lattuada et al., 2012).

\subsection{El concepto de agricultura familiar}

Coincidimos con Schiavoni en que "la AF en Argentina es una categoría en construcción. Su definición y sus alcances son objeto de múltiples negociaciones en las que intervienen científicos sociales, técnicos, administradores y organizaciones agrarias" (Schiavoni, 2010, p. 43, cursivas nuestras) [...] "La génesis de la AF en Argentina exhibe el carácter diverso de los productores que se aspira a unificar" (Schiavoni, 2010, p. 56).

El reconocimiento de la necesidad de conceptualizar desde el Estado este sector conllevó que la tipificación de la categoría se basara en dos factores: superficie reducida de tierra y uso de mano de obra familiar. Estos elementos condicionaron los criterios de selección de beneficiarios de los programas y proyectos de desarrollo rural en Argentina durante los años `90, enfatizando la pobreza y su inserción periférica en la economía (Schiavoni, 2010).

Si bien no entraremos aquí en el intenso debate sobre este concepto, articulando diferentes autores lo definimos como un tipo de producción en la cual la unidad doméstica y la unidad productiva están físicamente integradas. La agricultura es la principal ocupación y fuente de ingresos del grupo familiar y la familia aporta la fracción predominante de la fuerza de trabajo. La racionalidad del productor familiar es muy diferente a la lógica empresarial, ya que tiene como finalidad la reproducción de las unidades domésticas, distinguidas por el perfil familiar de la unidad, la fuerza del trabajo familiar, la mercantilización parcial de la producción, la indivisibilidad del ingreso familiar, la preferencia por tecnologías intensivas en mano de obra y la pertenencia a un grupo territorial; siendo en su interior que se realiza la transmisión de 
valores, prácticas y experiencias (Schejtman, 1983; Schiavoni, 2010; Feito, 2014; Obschatko et al., 2006; Foro de Organizaciones Nucleadas de la Agricultura Familiar, 2006).

Partiendo de las relaciones de producción, se puede establecer una tipología de unidades fundamentada en la combinación tierra/trabajo familiar y la incorporación de excedente traducido en capital y compra del trabajo asalariado (Murmis, 1998). Obschatko et al. (2006) caracterizan a los pequeños productores como aquellos que trabajan directamente en su predio, que no contratan mano de obra extra predial en forma permanente, introduciendo criterios de recorte según cantidad de superficie de la explotación, superficie máxima cultivada y posesión de unidades ganaderas dependiendo de la zona. Para el Foro de Organizaciones Nacionales de la Agricultura Familiar (FONAF) ${ }^{1}$, la AF es "una "forma de vida" y "una cuestión cultural", que tiene como principal objetivo la "reproducción social de la familia en condiciones dignas", donde la gestión de la unidad productiva y las inversiones en ella realizadas es hecha por individuos que mantienen entre sí lazos de familia, la mayor parte del trabajo es aportada por los miembros de la familia, la propiedad de los medios de producción (aunque no siempre de la tierra) pertenece a la familia, y es en su interior que se realiza la transmisión de valores, prácticas y experiencias" (Foro de Organizaciones Nucleadas de la Agricultura Familiar, 2006, p. 9, comillas en original). Tomamos como punto de partida la definición acordada por los países del MERCOSUR: "La pequeña agricultura familiar es un tipo de producción donde la unidad doméstica y la unidad productiva están físicamente integradas. La agricultura es un recurso significativo en la estrategia de vida de la familia, la cual aporta la fracción predominante de la fuerza de trabajo utilizada en la explotación. Y la producción se dirige tanto al autoconsumo como al mercado" (Foro de Organizaciones Nucleadas de la Agricultura Familiar, 2006).

Schneider (2003) reconoce que los análisis recientes sobre temas relacionados a las sociedades rurales y agricultura, tienen al marxismo como una de las vertientes analíticas que más se dedicó a estos objetos, especialmente en relación al análisis más específico sobre agricultura familiar y las formas sociales de trabajo vigentes en el mundo rural. Este autor asegura que el enfoque neomarxista percibe la pluriactividad o la opción por las actividades no agrícolas como una posibilidad de afirmar esa forma social agricultura familiar, en la estructura agraria del capitalismo contemporáneo. Afirma que los clásicos se interesaban por lo que hoy se denomina "agricultura familiar", a medida que éstos tenían un papel político a desempeñar como clase en proceso de desarrollo del capitalismo. Analizar la pluriactividad a partir del ambiente familiar, focalizando aspectos como la organización del proceso productivo, las formas de uso y alquiler de la forma de trabajo familiar o la inversión de destino de los arrendamientos y las relaciones de género, es un esfuerzo para demostrar que aunque la forma familiar sea afectada en cierto modo o condicionada por el mercado o por la dinámica capitalista de producción, esta forma tiene capacidad para adaptarse y establecer estrategias que garanticen su reproducción social (Schneider, 2003).

Por otra parte, un análisis cualitativo comprende esquemas de percepción que explican prácticas de reproducción social de la AF, mostrando cuestiones recurrentes en todo el país, tales como: tendencia a no residencia rural; necesidad excluyente de contar con trabajo extrapredial para subsistencia del grupo familiar; envejecimiento de productores que aún continúan en actividad; arrendamiento de fincas propias para asegurar ingresos y deterioro del parque de herramientas (Indelangelo et al., 2013).

La llamada "otra agricultura" (o, en términos de Pedro Peretti, 2013), "lo que queda") consiste en un mundo de personas plural y heterogéneo que se reconocen como productores familiares, minifundistas, campesinos, chacareros, colonos, medieros, granjeros y muchas otras denominaciones a lo largo y ancho del país. Son productores pequeños y medianos que no siempre son pobres, pero sí tienen dificultades de acceso a recursos esenciales (naturales como tierra y agua, pero también tecnología, información, financiamiento, asesoramiento técnico), que se encuentran produciendo y comercializando en desigualdad de condiciones frente a mercados cada vez más concentrados y exigentes.

\footnotetext{
1 Este Foro se transformó en 2014 en Federación de Organizaciones Nucleadas de la Agricultura Familiar, donde confluyen actualmente más de 900 organizaciones que asocian a unas 180 mil familias de productores en todo el país, siendo una de las organizaciones de tercer grado más importantes.
} 
Se requiere, como ya señalamos en otros trabajos (Feito, 2014), un apoyo diferencial para esta "otra agricultura". ¿Cómo se define este sujeto de políticas diferenciales? Regionalmente se está construyendo como una categoría política para poder focalizar políticas públicas de desarrollo rural (Lattuada et al., 2012). Entonces, la agricultura excede lo estrictamente agrícola y aún lo agropecuario, ya que involucra todas las actividades económicas llevadas en el medio rural (turismo, otros servicios, artesanías, agroindustria).

\subsection{Comercialización de la AF}

Podemos considerar la comercialización, siguiendo a Caracciolo $(2016$, p. 43) como el "proceso que va de la tranquera del productor hasta el comprador". La institucionalidad de apoyo a los procesos comerciales debería considerar un enfoque de tramas de valor, a fin de que las unidades económicas de la AF puedan desarrollar una gestión comercial que les permita generar valor agregado y satisfacer las necesidades de sus integrantes, al tiempo que ampliar sus emprendimientos en los mercados de manera sostenible (Caracciolo, 2016).

Para implementar procesos de desarrollo rural, son importantes las tramas institucionales conformadas por organismos públicos y privados dirigidas a organizaciones de pequeños productores para favorecer su inclusión económica y social. Estas tramas en general promueven participación política, posicionamiento en la cadena productiva, acceso a recursos (tierra y agua), gestión y ejecución de proyectos de infraestructura, mejoramiento de la producción. El avance en estos aspectos permite monitorear la conformación de una nueva territorialidad (Manzanal et al., 2009).

Por otro lado, los modelos informales de vinculación con los mercados son comunes pero poco comprendidos y no considerados en el apoyo de intervenciones para el desarrollo debido a su carácter informal y fuerte sesgo en contra de los comerciantes. Estos modelos tienen información y experiencias importantes para generar vínculos de mercados sostenibles y prestación de servicios, sobre todo allí donde la organización formal del agricultor es débil (Vorley et al., citado en Caracciolo, 2015).

Ploeg (2008) evidencia la resiliencia de los campesinos, en contrapunto a la noción hegemónica que no sólo los reduce a la condición de víctimas del capitalismo, sino que les usurpa el papel de sujetos de la historia. Evidencia que el campesinado, a pesar de haber sido mal entendido o insuficientemente estudiado, adentra el siglo XXI fortalecido en vista de la emergencia de lo que el autor denomina de Imperio, un modo de ordenamiento territorial global sostenido por estrategias de absorción de las riquezas producidas por otros. Hay, según el autor, tres dominios particulares que permiten comprender la naturaleza del Imperio alimentario: la agricultura, la producción y el consumo de alimentos y los esquemas reguladores a ellos asociados. La tesis sobre la que se mueve el autor es la de la recampesinización, definida como un proceso de transición que se desarrolla en varios niveles, a lo largo de varias dimensiones e involucrando a muchas personas.

El hilo conductor de su análisis es el modo de hacer agricultura, identificando tres sujetos distintos: el capitalista, el empresario y el campesino. El modo empresarial de hacer agricultura, referencia en el contrapunto establecido para construir la tesis de la recampesinización, estaría entre el modo capitalista, caracterizado por elevadas inversiones, gran escala de producción y contratación de fuerza de trabajo, y el campesino, que provenía de la condición campesina, que es distinta de los demás por estar fundada en intensificación del trabajo, en regla familiar, diversificación de la producción, baja disponibilidad de tierras y menor dependencia de recursos externos. En ese orden, lo que distingue el modo empresarial del capitalista sería la fuerza de trabajo familiar, no admitida en éste. Por su parte, para diferenciar al campesino del empresario no se invocan elementos diferenciales de clase, sino la dimensión de las propiedades y los niveles de incorporación de tecnologías exógenas. La situación de transición identificada por el autor lo hace vislumbrar en el modo empresarial de hacer agricultura una condición paradójica: al mismo tiempo que se plantea como principal amenaza al campesinado, por el imperativo de la expansión continua, también se presenta como campo fértil para la recampesinización, debido al descenso de la renta media en la actividad. 
Ploeg muestra que las redes que dan sustentación al Imperio son, en gran medida, virtuales, lo que no descarta su materialidad, por establecerse con un fin explícito: el control sobre la riqueza producida en las redes productivas de hecho. Esto impone el control de los puntos de distribución, de modo que para quien está fuera del Imperio, cada vez es más difícil vender. Allí se evidencia la importancia de los mercados locales, aunque es precisamente por la estandarización que el Imperio busca bloquearlos. La accesibilidad a la producción sería la palabra clave, por lo que cada vez más los productores tienen que pagar por el derecho de sus mercancías para llegar al consumidor. La potencial autonomía productiva, en vista de la capacidad singular de movilizar recursos en su favor, es lo que hace promisorio al modo campesino de hacer agricultura. La diferencia con la agricultura empresarial, es que ésta en lugar de potenciar los atributos de la naturaleza (como ocurre con la agricultura campesina), necesita someterla, ya que la variabilidad de los ciclos naturales de la agricultura dificulta la estandarización del proceso de trabajo, creando obstáculos a los incrementos de productividad. Por eso, la tónica de la agricultura empresarial es la artificialización, fundada en la incorporación de insumos externos, racionalización del trabajo, ingeniería genética, entre otros. Esto supone no sólo intensificación del capital en detrimento del trabajo, sino desconexión con los ecosistemas, resultando en pérdida proporcional de la eficiencia. Esto implica escalas de operación necesariamente opuestas: mientras que para el horizonte empresario es el mercado-mundo, para el otro, son los "circuitos cortos", es decir, los mercados locales. Entiende, así, que el modo campesino de hacer agricultura difiere radicalmente del negocio porque en el primero prevalece la búsqueda de soluciones locales para problemas globales, las cuales se traducen en incremento de renta local. Se trata de una estrategia fundada en la diversidad erigida en la articulación entre la base material y la social circunscrita localmente. Para él, por sí solo, es insurgente a la lógica global homogeneizante. En suma, la artificialización del proceso de producción agrícola es indisociable del proceso de externalización que, a su vez, está directamente ligado al aumento de la dependencia. Mientras la tónica del modo campesino de hacer agricultura es la artesanía, en el empresarial el llamamiento primado está en el espíritu emprendedor. Esta es la razón por la cual en el primero la renta es buscada en la intensificación del trabajo, mientras que en el segundo ella está directamente relacionada al aumento de la escala de la producción (Ploeg, 2008).

Como señalamos, la categoría AF es sumamente amplia y tiene un sustrato teórico fundamental como es la presencia central del trabajo familiar en el predio/actividad agropecuaria/agroindustrial. Por ello los canales de comercialización y sus respectivas problemáticas varían de acuerdo al tipo de AF de que se trate. Para gestar experiencias alternativas de comercialización sean exitosas, se debe considerar el concepto de cadena global de valor, que refiere a las "relaciones entre empresas y a los mecanismos institucionales a través de los cuales se coordinan los factores no definidos por el mercado con respecto a las actividades en la cadena". Esta coordinación se logra a través de la fijación y la imposición de parámetros de producto y proceso que están a cargo de los actores en la cadena (Humphrey \& Schmitz, 2010, citado en Caracciolo, 2015). Según esto, la AF ocupa una posición asimétrica con relación al poder de negociación para llegar a los mercados, por su escasa o nula participación en la fijación de las condiciones de producción y comercialización. Esto se debe a su ubicación subordinada en las cadenas de valor, vinculadas a su vez con cuestiones de escala o costos. Al estar la comercialización de alimentos altamente oligopolizada en las cadenas de supermercados, no se reconocen las posibilidades de producción de cada territorio, se incorporan costos de traslado innecesarios en producción primaria, industrialización, distribución y comercialización. Por ello las empresas fijan precios con tasas de ganancias superiores a las del resto de la economía (Centro de Estudios Scalabrini Ortiz, 2014).

La importante, compleja y con tantas aristas, cuestión alimentaria, es una cuestión de Estado: es innegable su rol en políticas que permitan el acceso de la totalidad de la población a los alimentos sanos, a precio justo, así como reconocer el ineludible el rol que cumple la AF como proveedor de alimentos sanos, de calidad, y en mercados de proximidad, por ello la importancia de las ferias en general y las realizadas en universidades en particular.

El acceso a una alimentación saludable es un aspecto decisivo para el bienestar de una sociedad. Sin embargo, muchas veces se dificulta por diversas cuestiones. La principal y que a su vez determina casi todas las demás, es la incidencia de la gran industria sobre la 
producción de alimentos, cuya lógica es fundamentalmente la obtención de la mayor ganancia en el menor tiempo posible. "No nutrir, no cuidar, ni siquiera ser saludable: simplemente ganar lo que más se pueda" (Barruti, 2013, p. 11 citado en Battista, 2016). Las cadenas concentradas de comercialización determinan los precios de los alimentos, en relación con la aceleración del proceso inflacionario argentino.

Los conceptos de cadenas cortas y redes agroalimentarias otorgan herramientas para repensar los mercados agroalimentarios y las relaciones entre consumidores y productores. Se destaca la importancia de la agricultura familiar en el proceso de creación de las cadenas cortas de comercialización, dado que los consumidores se benefician con las ferias orgánicas y agroecológicas, como forma de incentivar y valorar a los agricultores familiares al tiempo que los consumidores tienen la garantía de acceso a productos saludables. Las discusiones sobre las redes agroalimentarias, estimulan y difunden la idea de que el consumo de los alimentos sanos y producidos de forma sostenible pasa a tener más mercado, lo que acerca al agricultor $\mathrm{y}$ al consumidor. Se requieren nuevas estrategias de abastecimiento y cambiar la relación con los consumidores, lo que puede ser proporcionado por las cadenas cortas de comercialización de alimentos. El poder público, el mercado y la sociedad civil son protagonistas importantes en este proceso de reinventar modelos de comercialización. Cada vez más los consumidores quieren saber de dónde vienen los alimentos consumidos y de qué forma se producen. Esto también valora la producción de la agricultura familiar. Las cadenas cortas tienen un rol indispensable en la forma de organización de los mercados y permiten mejorar tanto la renta de los productores como las condiciones de alimentación de la población. También constituyen espacios de organización de la producción y comercialización de la agricultura familiar y otorgan alternativas al desarrollo rural (Gazolla \& Schneider, 2017).

Estos canales cortos de comercialización tienen las siguientes ventajas: generación de dinamización de las economías locales conectando productores y consumidores, ya que el dinero permanece en el circuito local; permite la participación de mujeres porque se desarrollan en espacios de proximidad, reducen el consumo de energía por la cercanía de los productos al espacio del mercado. Todo esto conlleva que el desarrollo local contribuya al mismo tiempo, al desarrollo nacional (Caracciolo, 2016).

Los alimentos de la AF no suelen llegar a los consumidores del mismo sector popular, porque compran en las grandes empresas. Incluso los mismos productores de la AF terminan comprando sus alimentos en las mismas. La intervención del Estado en este sector de la economía popular (mediante subsidios, asignaciones, políticas de redistribución del ingreso) mejora las posibilidades de consumo de sectores populares, pero constituye a su vez y paradójicamente, transferencias hacia el capital concentrado, porque fomenta el consumo en las grandes cadenas supermercadistas. Las relaciones de fuerza entre los actores en un contexto dado, modelan en cierto modo la regulación por parte del Estado de la tendencia hacia esta concentración económica.

Las dificultades de la AF para insertarse en los mercados, dada su heterogeneidad y desigualdades existentes en ellos, puede deberse a causas productivas, costos, logística, calidad, aspectos bromatológicos, impositivos, institucionales, financieros, etc. Si consideramos los mercados para la AF no debemos descuidar otros tipos de intercambios entre agentes económicos como la reciprocidad, distribución, etc. Si bien los mercados son centrales en economías complejas como la argentina, es posible pensar en otros mercados más justos y menos concentrados (Caracciolo, 2016) y que fomenten la soberanía alimentaria como eje de una estrategia de desarrollo que pretenda ser sostenible. La Vía Campesina plantea ante la "Cumbre de los Pueblos" la soberanía alimentaria como "...el derecho de cada pueblo y de todos los pueblos a definir sus propias políticas y estrategias de producción, distribución y consumo de alimentos, a fin de garantizar una alimentación cultural y nutricionalmente apropiada y suficiente para toda la población" (Carballo, 2017). 


\section{Resultados}

\subsection{Las ferias de la AF en Argentina como modalidad alternativa de comercialización}

Definimos las "ferias de la AF" como experiencias de provisión de productos de este sector agropecuario, sobre todo alimentarios, perecederos, de consumo masivo, y que son espacios valorados por los consumidores para proveerse de alimentos de la canasta básica (Goldberg \& Dumrauf, 2010).

Las ferias y mercados locales de la AF han constituido históricamente una de las estrategias de comercialización más efectivas para los pequeños productores. Constituyen canales alternativos de comercialización dentro del mercado interno, aportan a la diversificación de la comercialización, a la soberanía y seguridad alimentaria, contribuyen al desarrollo rural y de los territorios, promueven mejoras en los sistemas productivos y alternativas para el agregado de valor en origen. Los intercambios generados constituyen una oportunidad para el mejoramiento de las producciones al facilitar la conjunción de saberes entre los feriantes.

Estos espacios comerciales surgieron en el país como consecuencia de las políticas neoliberales y la desindustrialización iniciada a partir del proceso militar de 1976. El impacto de estos sucesos en el aumento de los índices de precarización, vulnerabilidad social y pobreza, se agudizaron con los procesos de Reforma del estado de la década de los años 90. Los procesos de privatización, la transnacionalización monopólica y la concentración latifundista, agravaron la crisis productiva y social (Alcoba \& Dumrauf, 2011). Estas ferias crearon un espacio alternativo propio de la AF para responder a sus problemas de comercialización, cuyo principal objetivo fue lograr contacto directo de productores con consumidores, pero sobre todo, contribuir al a mejora de la calidad de vida de la AF (Goldberg \& Dumrauf, 2010). En el período 1991/2001, aparecieron diversas experiencias cuyo papel fue significativo para comprender el proceso de formación de las ferias como estrategia del sector para superar la exclusión (Alcoba \& Dumrauf, 2011).

En las Ferias, el control bromatológico de los productos está a cargo de los gobiernos municipales, y en los últimos años, algunos grupos de feriantes organizados junto a diferentes instituciones avanzaron en la creación de sistemas participativos para garantizar la calidad de los alimentos. Se calcula que existen cerca de 600 ferias en todo el país, con mayor desarrollo en el noreste argentino y organizaciones de feriantes en varias provincias: Misiones, Corrientes, Formosa, Chaco, Santa Fe y Buenos Aires. Las ferias francas constituyen un elemento reactivador de las economías locales, y son promotoras de la soberanía alimentaria a través de la venta de alimentos frescos, sanos y naturales (Goldberg \& Dumrauf, 2010).

Esta forma de comercialización aparece favoreciendo un proceso de desarrollo sustentable, al propiciar lazos de solidaridad entre productores y consumidores. El propio ejercicio de las actividades, hace necesario resolver cuestiones diversas en términos socio-organizativos. La variedad de la oferta y la mayor diversidad de producciones que tienen cabida en las ferias y mercados locales, facilita acceder a una alimentación de mayor calidad (con mayor variedad, mejores cualidades nutritivas, más saludable y a precios justos). Al ser los tramos más cortos en la cadena producción- comercialización, mejoran las condiciones para identificar demandas y posibilidad de readecuar la oferta sobre la base de los requerimientos del consumidor, lo que a su vez realimenta la posibilidad de aumentar diversidad de las producciones y rescatar productos y saberes. Al acortarse las distancias, disminuyen los costos de transporte y al incrementarse las oportunidades para el agregado de valor a nivel local, la renta de los productores aumenta, incidiendo en la economía local. La generalización de estas experiencias repercute en la creación y consolidación de lazos sociales y sentido de pertenencia y valorización de la comunidad local. Como experiencias alternativas a los circuitos masivos, poseen potencial democratizador y distributivo que en tramos convencionales no se producen. Estas ferias se suelen emplazar en territorios donde la relación productor-consumidor es cotidiana, las familias se conocen a priori más allá del espacio de la feria, y hay confianza en la calidad de los productos que se comercializan (Caracciolo, 2015).

Mercedes Caracciolo realizó una tipología de nueve modalidades alternativas que vinculan a la producción, comercialización y consumo de alimentos frescos y procesados por fuera de la 
lógica del capital, considerando la satisfacción de las necesidades de productores y consumidores y a una distribución equitativa del valor agregado entre los diferentes actores, en lugar de buscar maximizar una tasa de ganancia sobre el capital invertido (Caracciolo, 2016).

Una de estos tipos lo constituyen estas "Ferias del Productor al Consumidor", que se desarrollan en general en espacios públicos ofreciendo los productos, principalmente hortalizas, a los consumidores de un cierto barrio o localidad. Funcionan, libres del pago de impuestos municipales, en general una vez por semana.

La feria de Unlam se enmarca en lo que Caracciolo (2016) denomina "Ferias y Mercados de relación directa Productor y Consumidor con gestión pública (Estado en alguno de sus niveles, y Universidades)". Esta modalidad intenta avanzar articulando productores y consumidores e incidiendo en las políticas públicas dentro y fuera de la Universidad, a través de una relación estrecha entre las áreas de docencia, investigación y extensión, a la vez que propone continuar generando articulaciones con otras organizaciones y programas de distintas instituciones públicas y privadas, intercambiando saberes entre productores, extensionistas, docentes, alumnos y además, intentando crear un Sistema Participativo de Garantía para incorporar prácticas agroecológicas en las fincas de los productores feriantes. Los productores consolidan sus procesos de fortalecimiento organizacional y se empoderan para lograr incidir en las políticas públicas, como informantes y como actores partícipes y decisores (Caracciolo, 2016).

\subsection{Transitando un camino: origen y desarrollo de la feria Unlam}

En los últimos años, en las instituciones científico-académicas se ha iniciado un proceso de reflexión acerca del papel de la ciencia y la tecnología en la resolución de algunos problemas estructurales de la sociedad argentina. No obstante, las universidades aún no logran institucionalizar espacios de participación a fin de incorporar las demandas de la sociedad y construir agendas para la generación de conocimientos para la resolución de problemas socioeconómicos y ambientales. En momentos en los que socialmente va profundizándose la conciencia y la aspiración por una vida más sana y una sociedad más solidaria y democrática, y con el objetivo de integrar las problemáticas de la AF a los objetivos educativos de las universidades, promover y concretar espacios alternativos de comercialización para este sector es una oportunidad para el intercambio de saberes entre productores de alimentos, técnicos de apoyo, docentes, no docentes, estudiantes y comunidad local en general.

La feria que analizamos surgió por la articulación y trabajo conjunto en terreno existente desde 2009 entre Unlam y EEA Amba Inta con actividades conjuntas de relevamientos en territorio basados en investigaciones internas de la universidad, de las cuales surgieron nuevas propuestas como negociación de un convenio de vinculación tecnológica que incluye al Instituto de Investigación para la Agricultura Familiar (Ipaf) Región Pampeana del Inta. En septiembre 2015 se aprobó la propuesta en Rectorado, comenzando a trabajar con el completo apoyo del Prosecretario de Extensión Universitaria y concretándose ocho ediciones de la feria, entre noviembre de 2015 y septiembre de 2017 (una de ellas realizada en el marco de la Bienal de Arte, un importante evento cultural y comunitario organizado hace varios años por la Universidad). Las fechas se deciden de acuerdo a las disponibilidades de espacio y recursos humanos en la universidad, por lo cual no tienen regularidad fija. Cada edición dura dos días consecutivos, en horario de 10 a 18 hs, comprometiéndose los feriantes a permanecer en sus puestos durante toda la feria. Se realizaron numerosas reuniones entre técnicos de Inta Amba y docentes investigadores de Unlam, e internas entre autoridades Unlam y equipo organizador (parte del cual formado por el Instituto de Medioambiente), así como seis reuniones generales incluyendo a las organizaciones de productores participantes. Se constituyó un Reglamento de funcionamiento; se desarrolló una estrategia de difusión a través de las respectivas prensas de Unlam e Inta Amba, (incluyendo al Centro de Estudiantes Unlam), se realizó en la primera edición una serie de talleres, y en la segunda, una encuesta a consumidores ya publicada (Jager, 2017); se dictó en la universidad un curso gratuito de Manipulación de Alimentos en agosto 2016 y otro de Formación de Costos en septiembre 
2016. Se conformó una entidad colectiva superadora de las diferencias entre organizaciones participantes, el grupo: "productores en la Unlam".

\subsection{Caracterización de los productores participantes y los productos presentados}

En las distintas ediciones de la feria, participaron un promedio de cuarenta feriantes, ofreciendo productos como: verduras, plantas de vivero, cactus, yerba, hongos frescos y secos, blends de té, pastas caseras, arándanos y derivados, mermeladas y dulces, miel y derivados, panificados, patio de comidas (tartas, empanadas, food truck), quiosco saludable, hilados, velas y sahumerios artesanales, artesanías con objetos naturales, aromáticas, aceites esenciales. Estos productos abarcan la casi totalidad de productos ofrecidos en primer lugar en las ferias a nivel nacional (Goldberg \& Dumrauf, 2010) (exceptuando los lácteos y derivados, carnes y embutidos y bebidas alcohólicas, cuya comercialización está prohibida en la Universidad). En ninguna feria nacional se venden ni ofrecen "servicios".

La cantidad y variedad de productos ofrecidos en la Feria Unlam fue en aumento a medida que mejoró la comunicación y divulgación de la feria, además del éxito reconocido por los feriantes en sus ventas y la conformidad de los participantes. Ellos mismos fueron invitando a otros feriantes a participar del proyecto. Varios feriantes provienen de la feria Itu-Amba que organiza hace más de un año el Municipio de Ituzaingó conjuntamente con la EEA Amba en el predio de la misma, todos los días viernes y un sábado por mes. Una de las consecuencias de la suma constante de feriantes de aquella feria, a medida que pasaban las distintas ediciones de la Feria Unlam, fue que en la Universidad debimos cambiar los días de semana de realización, a fin de no superponernos con la Feria Itu-Amba y permitirles a los feriantes participar de ambas (Feito, 2017).

El tipo de productos depende de los mecanismos de certificación bromatológica, por eso hay productos que son difíciles de ofrecer en cualquier feria del país, como los de origen pecuario.

Se confeccionó una Ficha de Inscripción que debieron completar y firmar todos los feriantes, que incluía información sobre sus datos personales (documento, nombre, domicilio, teléfono, correo electrónico o red social); los productos y su elaboración (rubro general, domicilio de elaboración, si es elaboración familiar o no, si el producto está inscripto o registrado y en cuál institución) y si participa en alguna organización y/o en otras ferias. Exclusivamente a los productores regionales se les preguntaba la organización o feriante que se hace cargo de la venta en la Feria.

La participación en la feria está condicionada, de acuerdo a lo determinado en las reuniones generales con los feriantes, en primer lugar, a ser productor local del distrito; segundo: si no es del distrito, debe ofrecer productos que no se producen localmente; tercero: si no es del distrito y los productos que ofrece ya son producidos localmente, debe esperar en lista de acuerdo a la cantidad de productores locales que se presenten, para no competir con ellos directamente. Este "orden" de incorporación permite privilegiar las producciones locales, con el objeto de en el mediano plazo, ir incorporando otros productos y también productos de otras regiones del país (en este caso, la condición para ingresar como feriante es producir uno mismo el producto y viajar a venderlo en la feria, o enviar a otra persona, familiar o no, en su representación; es decir, que no aceptamos productos de reventa). En el futuro estamos considerando la posibilidad de traer productos que se comercializan a través de cooperativas locales, que a su vez traen a la ciudad productos elaborados por otras cooperativas y organizaciones del interior del país a las cuales les es imposible ubicar sus productos si no fuera por estas comercializadoras solidarias.

En la tercera edición de la feria (llevada a cabo los días 11 y 12 de octubre de 2016), se confeccionó una Ficha de Información para la comercialización de productos de la AF que debieron completar todos los feriantes, conteniendo las siguientes variables: producto y sus especificaciones; información del productor u organización de productores (lugar donde produce, si es producción familiar, si está asociado, si tiene acompañamiento del INTA, etc.); presentación del producto (si es a granel, si viene fraccionado y peso, etc.); volumen de la oferta (si es estacional o no y en qué meses se ofrece, o si es una oferta anual continua); 
posibilidades de transporte a larga distancia; precio (aproximado y si hay diferencia entre precio mayorista y minorista). A esta ficha se adjuntaba foto del producto y los productores.

Realizamos trabajo de campo etnográfico en el contexto de esta feria, utilizando metodología cualitativa consistente en la realización de entrevistas en profundidad a la casi totalidad de feriantes (para obtener información primaria sobre elementos tales como: lugar de origen del feriante, residencia actual, tipo de producto ofrecido, cómo lo produce, tipo de mano de obra que utiliza, si agrega valor en origen, cómo conforma los precios, cómo es su relación con los clientes/consumidores, etc). También se realizó observación participante en el marco del desarrollo de la feria, para relevar interacción entre feriantes y clientes, las actitudes y tipos de consultas de los alumnos de la universidad que se acercaban a los puestos.

\subsection{Relevamiento de los consumidores en la feria}

En los últimos años se viene produciendo una creciente desconfianza ciudadana en torno a la alimentación (Calle Collado, Soler Montiel, Vara Sanchez, 2009; citado en Aguero et al., 2015). Este proceso consiste en la desconfianza de quienes comen y no producen su propia comida, y dependen de un complejo sistema agroalimentario, industrializado y globalizado que les ofrece a través del mercado, alimentos estandarizados y anónimos, de los cuales no tienen idea de dónde provienen ni quiénes los producen.

Con la intención de proveer herramientas a los feriantes para mejorar la atención a sus clientes, así como para desarrollar capacitaciones a los consumidores sobre qué y cómo comprar los alimentos que comen, implementamos a partir de la Segunda Edición de la Feria una encuesta a los consumidores, para poder caracterizarlos tomando distintas variables.

El cuestionario de encuesta fue elaborado y conducido durante el desarrollo de la Segunda edición por investigadores del equipo del Instituto de Medioambiente de la Unlam, sumándose en la tercera edición investigadores del Departamento Ciencias Económicas de la Universidad. El mismo consistió de preguntas abiertas y cerradas, considerando las siguientes variables: lugar de procedencia; composición etaria; cómo se enteró de la feria; nivel de escolaridad; categoría ocupacional; frecuencia de compra en las distintas ediciones de la feria (si estuvo en ferias anteriores); cantidad de productos que compró; valor de lo que compró; ingreso familiar; medio de traslado a la feria; tiempo de permanencia en la misma (en minutos); motivo de la compra; preferencias de compra (presentación del producto/ ubicación de la feria/percepción nivel higiene/trato de feriantes/existencia de productores familiares/variedad de producto/calidad de productos); cantidad de puestos en feria (adecuada o no); disposición a pagar por certificación; actividades a incorporar a la feria.

Los resultados muestran que la mayoría de los consumidores de la feria aún son estudiantes de la Universidad que se acercan por curiosidad o por previo conocimiento de ediciones anteriores. Lentamente se van acercando familiares de los alumnos, vecinos y amas de casa del barrio, a medida que aumenta la publicidad "boca a boca" y la difusión extra local a través del multimedia de la Universidad. Entre los consumidores, se privilegia la cuestión de la venta de productos alimenticios, ya sea en los productos que compraron como en los que hubieran querido comprar y no había. Por otra parte, la mayoría de los encuestados era gente joven (el estudiantado) y mujeres. Sería interesante tener en cuenta esta cuestión para incorporar en futuras ediciones de la feria alguna estrategia para captar otras edades y género. Así mismo, considerar poder llegar a otros partidos a través de otros medios, ya que la mayoría de los visitantes era proveniente de La Matanza. Un 17\% fue a la UNLAM exclusivamente a la feria. La mayoría toma la feria como un "paseo" y declaró haber concurrido para comprar "productos sanos". Las razones que prevalecieron para realizar la compra de productos son: la calidad y la naturalidad de los productos. Un $25 \%$ quiere que la feria incorpore otras actividades además de la venta de productos, tales como "juegos". Aquellos que respondieron que realizan compras en otras ferias, se proveen de productos como frutas, verduras, quesos y dulces. La gran mayoría valoró positivamente el trato de los feriantes hacia los compradores. Se enteraron de la existencia de la feria en su mayoría por el "boca a boca" (Jager, 2017). 


\section{Discusión}

\subsection{Acceso a la alimentación en Argentina}

El acceso a los alimentos es hoy en Argentina un tema de preocupación generalizada en la sociedad de consumo, máxime considerando las nuevas líneas neoliberales orientadoras de la economía, que desvalorizan la producción y distribución de alimentos sanos y de proximidad. Prueba de ello es el intento de desmantelamiento progresivo por parte de la actual gestión de gobierno iniciada en diciembre de 2015 de organismos públicos directa o indirectamente relacionados con la alimentación en el Ministerio de Agroindustria de la Nación (Minagro), como la Secretaria de Agricultura Familiar que fue disminuida a categoría de Subsecretaría, así como la eliminación de la Dirección Nacional de Fortalecimiento Institucional y Apoyo a las Organizaciones.

La mejora en el acceso a los alimentos para todos los habitantes del país debería ser una política de Estado privilegiada y de primer orden. Por el contrario, se promueven las exportaciones alimentarias y la apertura de negocios hacia todo el mundo descuidando el precio de los alimentos para la población local ${ }^{2}$. Es decir la producción de alimentos es vista exclusivamente como un agronegocio y no como una actividad económica que debe proveer de alimentos sanos, de calidad y a precios accesibles a toda la población. Otro desacierto es la reformulación de uno de los principales programas de desarrollo rural del país, Cambio Rural, creado en el año 1993 y modificado en el año 2015. Este programa fue durante más de veinte años una referencia del accionar del Estado para la organización y asistencia técnica hacia las pequeñas y medianas empresas agropecuarias, otorgando diversidad de estrategias de fortalecimiento organizacional, mejora de manejo, buenas prácticas agropecuarias y de comercialización y tecnologías apropiadas, que permitieron mejorar sus producciones, su comercialización y su calidad de vida. La Resolución 249 E del 6 de septiembre de 2017 del Minagro, muestra en el punto 5.1 del Manual Operativo, los siguientes requisitos para los nuevos beneficiarios: "ser nacido en territorio argentino, o ser hijo de ciudadano nativo", "ser productor pequeño o mediano del sector agropecuario o agroalimentario", "desarrollar la actividad productiva y/o de servicios con carácter exclusivamente comercial", "reunir un ingreso anual neto para sí, superior al 50\% de los ingresos totales". Esto excluye a la gran mayoría de productores hortícolas del país y sus hijos, generalmente bolivianos con status migratorio legal, quienes garantizan el abastecimiento de las grandes ciudades del país, aportando seguridad alimentaria a la población. Asimismo se excluyen de uno de los principales programas del sistema nacional de desarrollo y extensión rural a los productores familiares, quienes no producen "exclusivamente para comercialización" sino, como veremos más adelante, en la mayoría de los casos también para autoabastecimiento.

\subsection{La AF en el contexto agropecuario nacional: necesidad de políticas diferenciales}

La AF en Argentina no es únicamente agrícola y a la vez, contribuye con servicios ecosistémicos, en el poblamiento del territorio, la oferta diversa de alimentos tradicionales y protección de biodiversidad, cultura y soberanía alimentaria.

La centralidad es que su modo de hacer en la práctica como su relación con el mundo rural, los conforma de manera diferente, tanto como que los sujetos a cargo de las actividades económicas rurales no son todos iguales. No es lo central la familia ni aún la agricultura. Se trata de una categoría política, por su acceso diferencial y asimétrico a la información, recursos y poder, de estos sujetos de la "AF" y los de la gran agricultura o agricultura empresarial o patronal (Lattuada et al., 2012). Se incluye aquí tanto a pequeños productores

\footnotetext{
${ }^{2}$ Esto se ejemplifica con la próxima realización en Argentina de la primera exposición alimentaria B2B de primer nivel y ronda internacional de negocios alimentarios de Latinoamérica, "Aliment-Ar", apoyada por el Minagro, cámaras binacionales y sectoriales, representantes de las cadenas de valor más significativas y embajadas de veintiún países latinoamericanos participantes. Esta mega exposición desestima la producción familiar, siendo pensada para exportadores, distribuidores, compañías de alimentos y Pymes argentinas y latinoamericanas, con la idea de que "Argentina puede ser el supermercado del mundo" (Clarín Digital, 2017).
} 
minifundistas, como a diferentes estratos, de reproducción deficitaria, simple o ampliada del sector rural, que, todos juntos, compiten con la agricultura empresarial.

¿Cómo se materializa esto en el contenido de las políticas diferenciales? Esto depende de la concepción política en la que se inscriba ese acuerdo sobre AF. Existen dos abordajes que se corresponden con nociones del desarrollo: "hay políticas que se basan en aspectos "sociales", orientadas hacia asistencia con la idea de compensar desigualdades y políticas que se centran en aspectos "económicos", que devienen en carácter sectorial (fortalecer desarrollo rural mediante mecanismos que no distorsionen la producción y el comercio) (Lattuada et al., 2012, p. 82, comillas en original).

El modelo de desarrollo agrario actual sobre todo en la Región Pampeana (dónde se manifiesta hegemónico), asentado en la producción de bienes exportables, tecnología intensiva de insumos y capital, favorece a las economías de mayor escala con concentración económica y lesiona gravemente la base de la soberanía alimentaria de la población rural, periurbana y urbana (Centro de Investigación para la Agricultura Familiar del INTA, 2006). Sin embargo, la condición familiar otorgó a los pequeños productores flexibilidad para permanecer en la producción, por la capacidad de adecuar consumo e inversión a las oscilaciones de los ingresos, cambiando sus actividades productivas de acuerdo a la demanda internacional y las políticas nacionales. En el discurso dominante, la AF es identificada como un actor en decadencia (Foro de Universidades para la Agricultura Familiar Region Pampeana, 2011).

Pero a pesar del retroceso en términos cuantitativos, continúa siendo una forma de producción presente y necesaria en la actual estructura social agraria (Foro de Universidades para la Agricultura Familiar Region Pampeana, 2011). El 71\% de los productores agropecuarios pertenecen a este sector; en tanto el valor bruto estimado de la producción de estos productores representa el $19,2 \%$ del valor generado por el total de explotaciones agropecuarias. La producción por hectárea es $53 \%$ mayor en el promedio de estos pequeños productores que en los que no lo son, lo cual evidencia que los primeros presentan mayor productividad de la tierra. Este sector participa en un 19\% en la contratación de servicio de maquinaria agrícola. En relación al empleo, las EAPs del sector concentran el $87,3 \%$ del trabajo de familiares del productor, en tanto aportan el 53\% del empleo total (54\% del trabajo permanente y el $29 \%$ del trabajo transitorio directo) en el sector agropecuario a nivel nacional (Obschatko et al., 2006).

Los AF tienen la potencialidad para ofrecer a los mercados de todas las regiones de Argentina, alimentos frescos, con menor incorporación de conservantes y aditivos, con baja o nula exposición a agroquímicos, con mejor calidad y variedad en todas las estaciones del año. La acción del Estado es irreemplazable para transformar esta potencialidad en hechos, mediante la promoción de mejoras en los sistemas productivos y en asistencia para el desarrollo de producciones que permitan el agregado de valor en origen y una comercialización cercana a los lugares de producción. Sin embargo, los agricultores familiares deben enfrentar múltiples problemas que condicionan su actividad en lo referente a la producción, la circulación y la distribución de sus producciones (Foro de Universidades para la Agricultura Familiar Region Pampeana, 2011).

\section{Conclusiones}

La relevancia de la $A F$, sus potencialidades actuales y futuras para el sostenimiento de un proyecto de desarrollo con inclusión social y su papel destacado en la provisión de alimentos sanos para el conjunto de la población, contribuyendo a la seguridad y soberanía alimentaria, constituyen motivos suficientes para que las sedes de las universidades nacionales aporten espacio físico, logística y gestión, para la difusión y valorización de la actividad, a través de la realización de ferias periódicas de comercialización de sus productos en sus distintas sedes.

En el caso analizado, como obstáculos mencionamos: problemas de financiamiento (los feriante decidieron armar un pozo común de dinero con aportes propios regulares y el equipo organizador intenta gestionar fondos en la Universidad); dificultad para conseguir gazebos y armar los puestos; coordinación y logística; tensiones entre organizaciones participantes; 
problemas de formalización y de inocuidad y "legalidad" de los productos; dificultades para una certificación social participativa (no existe aún normativa del Servicio Nacional de Calidad y Sanidad Alimentaria que incluya la producción de este sector); dificultades para decidir qué tipos de productos vender; superar niveles de informalidad organizativa.

Como avances destacamos: comenzó un proceso de fortalecimiento organizacional, se superaron algunas diferencias entre organizaciones participantes en pos de la concreción de la feria, se realizaron varias capacitaciones (sobre Manipulación de Alimentos y Formación de Costos), se fortaleció la articulación Inta/Unlam.

Así, generar la oportunidad para el desarrollo de la feria es una muestra de que la universidad fortalece a los agricultores familiares como actores políticos al promover el asociativismo.

Asimismo, se intenta lograr cierta coordinación de los factores no definidos por el mercado respecto de las actividades a lo largo de la cadena de producción/comercialización, fijando e imponiendo parámetros de proceso y de producto a cargo de los actores de la cadena. Esto implica mantener el criterio de que sólo participen de la feria aquellos que realmente producen lo que comercializan, si bien no llegamos aún a tener control desde la Universidad del proceso de producción ni de la trazabilidad del producto desde la finca hasta el puesto en la Feria ${ }^{3}$.

Uno de los aspectos que posibilitarían la consolidación del proceso interno de la Feria es el de las capacitaciones, especialmente en temas como: gestión de la calidad (de productos y procesos), inocuidad, presentación de puestos, forma de fidelización de clientes; herramientas de comunicación; etiquetado y envasado; conservas (Aguero et al., 2015). Esto se refleja en la preocupación de los feriantes en la atención al cliente y en la defensa que realizan desde sus discursos, de la posibilidad concreta de aprovechar la oportunidad que les brinda la Universidad para comercializar en forma gratuita sus productos.

Estas ferias son un potente instrumento de comercialización de la AF y su crecimiento significativo en los últimos años revela la importancia estratégica de construir y/o fortalecer políticas públicas que afiancen su expansión. Para ello, se requiere fundamentalmente: fortalecer el rol del Estado en promoción y apoyo; profundizar la investigación sobre ferias y dinamizar el trabajo de extensión en el desarrollo de las mismas; visualizar e identificar nuevas estrategias de comercialización; construir normativas bromatológicas apropiadas; desarrollar estrategias de comunicación para promoverlas y vincular al productor con el consumidor; facilitar mecanismos de financiamiento para logística e instalación de esta iniciativa con una frecuencia regular.

Es importante destacar que este tipo de ferias en Universidades no tienen el objetivo final de las Ferias Francas que mostramos que se desarrollan a nivel nacional (si bien, como vimos, comparten muchas de sus características), ya que el mismo consiste en lograr la autogestión de los productores/feriantes, pero en el caso analizado, todas las decisiones que tome la organización como los feriantes, deben ser siempre ad referéndum de la decisión final de las autoridades universitarias, quienes ofrecen el predio en forma gratuita y sin ninguna obligación a cambio por parte de los feriantes, que hacen uso de las instalaciones especialmente puestas a disposición para ellos mediante el trabajo de los departamentos de Mantenimiento, Seguridad e Higiene, administración, que colaboran en facilitar la estancia de los feriantes durante todo el día en sus puestos: carros para transportar los productos, permiso de ingreso de vehículos al predio donde circulan personas; bidones de agua a disposición, sillas, limpieza, baños.

La feria analizada es un ejemplo de que este tipo de espacios alternativos de comercialización para la AF mejoran el desempeño de las unidades productivas, no sólo en resultados económicos dado el ingreso monetario por ventas, sino también en avances en los intercambios entre los actores que se producen a través de las experiencias y transacciones comunes, tanto en las etapas previas de preparación de la feria como en los días de su desarrollo en el predio de la Universidad. Las tramas de relaciones que se dinamizan a través

\footnotetext{
${ }^{3}$ Este "control" es legitimado en cierta forma por el Inta, dado que los técnicos que participan en la organización de la Feria analizada, son también técnicos de terreno en el distrito, y en general conocen las producciones y los productores participantes.
} 
de estos intercambios inciden en un proceso de desarrollo territorial inclusivo, que se refleja en un incremento del capital social, traducido en fortalecimiento de organizaciones e instituciones (Battista, 2016) y los aprendizajes y experiencias adquiridos por los alumnos que concurren en calidad de visitantes/consumidores/compradores, que en un mediano plazo, podrían incorporarse a las currícula de determinadas materias relacionadas con la producción y comercialización de alimentos en los distintos Departamentos y carreras.

La experiencia de la feria de la Unlam evidencia que la comercialización es un eje importante del desarrollo rural, pero destacamos que lo que falta es una política integral a nivel nacional de desarrollo rural para la agricultura familiar.

Fortaleciendo modalidades alternativas de comercialización de la AF, se opera una moderación de tendencias a concentración y transnacionalización del sector agropecuario, aceptando la importancia económica vital de este sector.

En definitiva, la problemática del desarrollo rural local tiene un eje importante en la comercialización.

\section{Referencias}

Aguero, D., Sandoval, G., Carrera, R., Freire, V., Crivellaro, M., Gramaglia, C., Dumrauf, S. (2015). La feria de Villa Las Rosas en Traslasierra, Córdoba. Córdoba: Ed. Universidad Nacional de Rio Cuarto.

Alcoba, D., \& Dumrauf, S. (Comps.). (2011). Del productor al consumidor. Apuntes para el análisis de las ferias y mercados de la Agricultura Familiar en Argentina. Bs As: Ediciones INTA.

Ander Egg, G. (1995). Técnicas de investigación social. Buenos Aires: Ed Lumen.

Battista, S. C. (2016). Las ferias y mercados de la Agricultura Familiar (AF) y su aporte al desarrollo territorial con inclusión social en el Periurbano Oeste Bonaerense (Informe de Avance Proyecto PROINCE). La Matanza: Universidad Nacional de La Matanza.

Caracciolo, M. (2015). Situación de la institucionalidad de apoyo a la innovación comercial y de los procesos de gestión comercial de la agricultura familiar en la Argentina. Bs As: IICA. Recuperado el 12 de septiembre de 2017, de http://www.iica.int

Caracciolo, M. (2016). Soberanía alimentaria y mercados alternativos (Monogafía). Programa en Economía Solidaria. Escuela de Economía y Negocios, Universidad Nacional de Gral San Martín, Centro de Estudios y Formación en Economía Social y Solidaria La Yumba, Cooperativa de Consumo Ltda, Buenos Aires.

Carballo, C. (2017). La soberanía alimentaria en Argentina. Avances, resistencias y propuestas. Revista Realidad Económica, (35), 92-117.

Centro de Estudios Scalabrini Ortiz - CESO. (2014). Economía Populary Trabajo (N 1). Buenos Aires: CESO.

Centro de Investigación para la Agricultura Familiar del INTA - CIPAF. (2006). La Juntada. Microcrédito, tecnología y gestión asociada en la Agricultura Familiar. Bs As: Ed INTA.

Clarín Digital. (2017, septiembre 22). El mundo de los alimentos se reúne en la Argentina: llega la exposición Aliment.ar. Argentina: Clarín Digital. Recuperado el 17 de septiembre de 2018, de http://www.clarin.com.ar/

Escobar, A. (1995). El desarrollo sostenible: diálogo de discursos. Ecología Política, 9, 7-25.

Esteva, G. (2000). Desarrollo. In A. Viola (Comp.), Antropología del desarrollo. Teorías y estudios etnográficos en América Latina. Barcelona: Editorial Paidós Studio.

Feito, M. C. (2017, Enero 6-13). Comercialización de la agricultura familiar periurbana como experiencia de extensión universitaria: la "Feria del Productor al Consumidor". In Actas 9 Congreso Chileno de Antropología. Argentina: Universidad Nacional de La Matanza.

Feito, M.C. (2014). Ruralidades agricultura familiar y desarrollo. Territorio del Periurbano Norte de la provincia de Buenos Aires. Bs As: Editorial La Colmena.

Ferguson, J. (1990). The anti-politics machine. 'Development", depoliticization and bureaucratic power in lesotho. Cambridge: Cambridge University Press.

Foro de Organizaciones Nucleadas de la Agricultura Familiar - FONAF. (2006). Documento Base del FoNAF para implementar las políticas públicas del sector de la Agricultura Familiar. Recuperado el 31 de octubre de 2018, de http://www.fonaf.com.ar/documentos/Docmento_base_FoNAF.pdf

Foro de Universidades para la Agricultura Familiar Region Pampeana. (2011). Documento presentación. Buenos Aires: Ed INTA. 
Gazolla, M., \& Schneider, S. (Orgs.). (2017). Cadeias curtas e redes agroalimentares alternativas: negócios e mercados da agricultura familiar. Porto Alegre: UFRGS Editora.

Geertz, C. (1992). La interpretación de las culturas. Barcelona: Ed. Gedisa.

Glasser, B. G., \& Strauss, A. L. (1967). The discovery of the grounded theory. Srategies for qualitative research. New York: Aldine Publishing Company.

Goldberg, C., \& Dumrauf, S. (2010). Agricultura Familiar: las ferias de la agricultura familiar en la Argentina. Buenos Aires: Ed. INTA.

Guber, R. (1991). El salvaje metropolitano. A la vuelta de la antropología posmoderna. Buenos Aires: Ed. Paidos.

Guber, R. (2001). Etnografía, método, campo y reflexividad. Buenos Aires: Ed Norma.

Indelangelo, N., Main, C., \& Prividera, G. (2013). La agricultura familiar en el departamento de Diamante (Entre Ríos). Una primera aproximación para su análisis. In D. Ramilo \& G. Prividera (Comps.), La Agricultura Familiar en la Argentina. Diferentes abordajes para su estudio. Buenos Aires: Ed. INTA.

Jager, M. (Comp.) (2017). Gobernabilidad, Percepción, Control y Efectos del Uso de Agroquímicos en la Región Metropolitana de Buenos Aires. Presunta contaminación por el uso inadecuado de agroquímicos. San Justo: Ed Universidad Nacional de La Matanza.

Kottak, C. P. (2000). Cultura y desarrollo económico. In A. Viola Recasens (Comp.), Antropología del desarrollo. Teorías y estudios etnográficos en América Latina. Barcelona: Editorial Paidós Studio.

Lattuada, M., Marquez, S., \& Neme, J. (2012). Desarrollo rural y política. Reflexiones sobre la experiencia argentina desde una perspectiva de gestión. Bs As: Ed Ciccus.

Manzanal, M. (2006). Regiones, territorios e institucionalidad del desarrollo rural. In M. Manzanal, G. Neiman \& M. Lattuada (Comps.), Desarrollo rural. Organizaciones, instituciones y territorios. Bs As: Ed Ciccus.

Manzanal, M., Arqueros, M. X., Arzeno, M., \& Nardi, M. A. (2009). Desarrollo territorial en el Norte argentino. Una perspectiva crítica. Revista Eure, 35(105), 131-153.

Martinez Carazo, P. (2006). El método de estudio de caso: estrategia metodológica de la investigación científica. Pensamiento \& Gestión, 20, 165-193.

Murmis, M. (1998). El agro argentino, algunos problemas para su análisis. In N. Giarraca \& S. Cloquel (Comps.), Las agriculturas del Mercosur, el papel de los actores sociales. Buenos Aires: Ed La Colmena.

Obschatko, E., Foti, M., \& Roman, M. (2006). Los pequeños productores en la república Argentina. Importancia de la producción agropecuaria y en el empleo en base al Censo Nacional Agropecuario 2002. Bs As: SAGYP/IICA.

Peretti, P. (2013). Chacareros, soja y gobernabilidad. Del Grito de Alcorta a la Resolución 125. Bs As: Ciccus.

Ploeg, J. D. (2008). Camponeses e impérios alimentares: lutas por autonomia e sustentabilidade na era da globalização (Trad. Rita Pereira). Porto Alegre: UFRGS. Recuperado el 17 de septiembre de 2018, de https://www.researchgate.net/publication/270857519_Camponeses_e_imperios_alimentares_lutas por_autonomia_e_sustentabilidade_na_era_da_globalizacao

Schejtman, A. (1983, Abril-junio). Campesinado y desarrollo rural; lineamientos de una estrategia alternativa. Investigación Económica, 42(164), 115-152.

Schiavoni, G. (2010). Describir y prescribir: la tipificación de la agricultura familiar en Argentina. In M. Manzanal \& G. Neiman (Comps.), Las agriculturas familiares del Mercosur. Trayectorias, amenazas y desafíos. Bs As: Ed Ciccus.

Schneider, S. (2003). A pluriatividade na agricultura familiar (2. ed., 252 p., Estudos Rurais Series). Porto Alegre: Editora da UFRGS. Recuperado el 17 de septiembre de 2018, de http://books.scielo.org/id/b7spy/epub/schneider-9788538603894.epub

Taylor, S., \& Bodgan, R. (1987). Introducción a los métodos cualitativos de investigación (2. ed.). Buenos Aires: Paidos.

Viola Recasens, A. (2000). La crisis del desarrollismo y el surgimiento de la antropología del desarrollo. In A. Viola Recasens (Comp.), Antropología del desarrollo. Teorías y estudios etnográficos en América Latina. Barcelona: Editorial Paidós Studio.

Data de submissão: 2 de novembro de 2017

Data de aceite: 22 de dezembro de 2018.

Classificação JEL: Q13, Q18. 